\title{
Estado nutricional infantil no concelho de Portalegre
}

Adriana Gameiro, ${ }^{1}$ Victória Garrett ${ }^{2}$

\section{RESUMO}

Objetivos: Determinar a prevalência de baixo peso, peso normal, excesso de peso e obesidade na população em idade escolar numa Unidade de Saúde Familiar (USF) do concelho de Portalegre e determinar se existe diferença estatisticamente significativa entre o género e o peso e entre a idade e o peso.

Tipo de estudo: Estudo observacional transversal, descritivo e analítico.

Local: USF Portus Alacer, concelho de Portalegre.

População: Utentes da USF Portus Alacer [6-17 anos].

Métodos: Determinação do índice de massa corporal (IMC) de 448 crianças e adolescentes [6-17 anos] vigiadas na USF Portus Alacer, entre janeiro de 2017 e outubro de 2018 [intervalo de confiança (IC) 95\%, margem de erro 3,64\%]. Os participantes foram classificados em quatro categorias de acordo com os pontos de corte de IMC estabelecidos pela OMS para a idade e género. O software usado no tratamento de dados foi o SPSS ${ }^{\circledR}$ v. 25.0, o Microsoft Office Excel ${ }^{\circledR}$ e o Sample Size Calculator by Raosoft ${ }^{\circledR}$. As ferramentas estatísticas utilizadas foram o teste de Qui-quadrado e o teste do $t$-Student. A normalidade foi testada através do teste Kolmogorov-Smirnov.

Resultados: Das crianças avaliadas 52,7\% eram do sexo masculino. A mediana etária foi de 12 anos. Verificou-se uma prevalência de peso excessivo de $37,4 \%$. A prevalência do excesso de peso foi de $16,7 \%$, superior no sexo feminino, e a da obesidade foi de $10,7 \%$, ligeiramente superior no sexo masculino. A maior prevalência de peso excessivo verificou-se aos sete anos. Não foram encontradas diferenças estatisticamente significativas entre o género e a categoria de peso, nem entre as idades relativamente ao peso excessivo.

Conclusões: Apesar da diminuição da prevalência da obesidade infantil e excesso de peso verificada em Portugal nos últimos anos, estas entidades representam um grave problema de saúde pública. No concelho de Portalegre, a prevalência do peso excessivo nos menores de 10 anos foi superior aos dados disponíveis na literatura. Dos 10 aos 17 anos a prevalência foi inferior.

Palavras-chave: Excesso de peso; Obesidade pediátrica; Índice de massa corporal.

\section{INTRODUÇÃO}

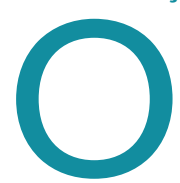

peso excessivo (excesso de peso e obesidade) em crianças e adolescentes constitui um importante problema de saúde pública. ${ }^{1-2}$ Se a atual tendência de obesidade infantil não for invertida até 2025, estima-se que mais de um em cada três adultos na Europa será obeso. ${ }^{3}$

A morbilidade associada a esta patologia acompanha inevitavelmente a diminuição da qualidade de vida e os custos elevados em saúde, ${ }^{2,4-5}$ constituindo uma preocupação para a comunidade científica e urge a necessidade de implementação de estratégias de prevenção e intervenção atempada. ${ }^{6-10}$

1. USF Portus Alacer, ULS do Norte Alentejano. Portalegre, Portugal. 2. USF Plátano, ULS do Norte Alentejano. Portalegre, Portugal.
O índice de massa corporal (IMC) em $\mathrm{kg} / \mathrm{m}^{2}$ constitui o parâmetro recomendado pela Organização Mundial da Saúde (OMS) para a avaliação do estado nutricional. ${ }^{2,4,11}$

Em Portugal, a Direção-Geral da Saúde (DGS) recomenda a utilização das curvas padrão de crescimento preconizadas pela OMS. ${ }^{4,12}$ Os pontos de corte adotados pela OMS definem o baixo peso para um IMC inferior ao P3, o peso normal para um IMC superior ou igual ao P3 e inferior ao P85, o excesso de peso para um IMC igual ou superior ao P85 e inferior ao P97, e a obesidade para um IMC superior ou igual ao P97.

O Childhood Obesity Surveillance Initiative (COSIOMS Europa) ${ }^{13}$ é um sistema de vigilância do estado nutricional infantil das crianças em idade escolar (entre os seis e os oito anos) e produz dados comparáveis 
entre países europeus, permitindo a monitorização da obesidade infantil a cada dois ou três anos. Atualmente, é o maior estudo europeu da OMS e descreve a nível nacional (COSI Portugal) uma tendência invertida nas prevalências de excesso de peso e de obesidade infantil.

De acordo com a quinta ronda do COSI Portugal $2019,29,6 \%$ das crianças pertencentes ao estudo revelaram excesso de peso e $12 \%$ obesidade infantil, verificando-se uma redução de $8,3 \%$ (37,9\% em 2008) e de $3,3 \%$ (15,3\% em 2008), respetivamente. ${ }^{13-14}$

A quinta ronda do COSI Portugal apresentou ainda resultados para o estado nutricional infantil por região do país. Relativamente aos resultados alusivos à região do Alentejo, os dados obtidos indicam que de 2016 para 2019 ocorreu um decréscimo de 3,2\% (de 27,1\% para $23,9 \%$ ) na prevalência do excesso de peso e de $2,5 \%$ (de $12,2 \%$ para $9,7 \%$ ) na prevalência da obesidade infantil. ${ }^{13-14}$

De salientar que a região do Alentejo apresentou a menor prevalência de obesidade infantil a nível nacional $(9,7 \%$ face a $12 \%)$ e a segunda menor prevalência de excesso de peso a nível nacional (23,9\% face a $29,6 \%)$. Em relação à prevalência de baixo peso na região do Alentejo, esta foi de $0,9 \%$ (face a $1,3 \%$ a nível nacional). ${ }^{13}$

Relativamente ao estado nutricional infantil por género, o COSI Portugal 2019 descreve uma maior prevalência de obesidade $(13,4 \%)$ e de excesso de peso $(29,6 \%)$ no sexo masculino relativamente ao sexo feminino (obesidade: 10,6\%; excesso de peso: $29,5 \%$ ). A prevalência de baixo peso foi ligeiramente superior no sexo masculino relativamente ao sexo feminino $(1,6 \%$ vs 0,9\%). À semelhança de rondas anteriores verificou-se que a prevalência de obesidade infantil aumenta com a idade. Em 2019 15,3\% das crianças com oito anos apresentavam obesidade, comparativamente às crianças com seis anos que apresentavam uma prevalência de obesidade inferior $(10,8 \%){ }^{13}$

Segundo o Programa Nacional para a Promoção da Alimentação Saudável de 2017, ${ }^{6}$ a prevalência de excesso de peso em crianças com idade inferior a 10 anos é de $17,3 \%$ e a prevalência de obesidade nesta faixa etária é de $7,7 \%$. Já na faixa etária dos 10 aos 17 anos, a prevalência de excesso de peso é de $23,6 \%$ e a prevalência de obesidade é de $8,7 \%$.
O presente estudo tem como objetivo determinar a prevalência de baixo peso, peso normal, excesso de peso e obesidade na população infanto-juvenil em idade escolar numa Unidade de Saúde Familiar do concelho de Portalegre, segundo os critérios da OMS,, ${ }^{4,12}$ e estabelecer uma comparação face ao panorama nacional. Pretende ainda determinar se existe diferença estatisticamente significativa entre o género e o peso excessivo (excesso de peso e obesidade) e entre a idade e o peso excessivo.

\section{MÉTODOS}

Efetuou-se um estudo observacional transversal, descritivo e analítico.

De um total de 1.168 crianças e adolescentes em idade escolar, dos seis aos 17 anos, inclusive, inscritas na USF Portus Alacer, foi obtida uma amostra de 448 crianças e adolescentes [intervalo de confiança (IC) 95\%, margem de erro $3,64 \%]$, avaliadas no âmbito da consulta de saúde infantil e juvenil entre janeiro de 2017 e outubro de 2018.

Os dados individuais (idade em anos e género) e antropométricos [IMC, segundo a fórmula peso $(\mathrm{kg}) / \mathrm{es}-$ tatura $\left.(m)^{2}\right]$ foram extraídos dos registos efetuados no processo clínico. O peso e a estatura para determinação do IMC foram obtidos pela equipa de saúde durante as consultas de saúde infantil e juvenil. Os dados foram extraídos pelas autoras no mês de outubro de 2018.

$O$ peso foi medido em balança mecânica de contrapeso (marca Jofre), com capacidade máxima de $150 \mathrm{Kg}$ e precisão de $100 \mathrm{~g}$. A estatura foi medida com estadiómetro. Para avaliação dos dados antropométricos as crianças estavam em posição ortostática, descalças e parcialmente vestidas (em roupa interior, sem sapatos e sem adornos). ${ }^{2}$

O projeto de investigação foi autorizado pela comissão de ética na Unidade Local de Saúde do Norte Alentejo.

Os participantes foram classificados em quatro categorias, de acordo com os pontos de corte de IMC definidos pela OMS: baixo peso (IMC < percentil 3), peso normal (percentil $3 \geq$ IMC $<$ percentil 85), excesso de peso (percentil $85 \geq$ IMC $<$ percentil 97) e obesidade (IMC $\geq$ percentil 97). ${ }^{4,12}$

Os resultados obtidos foram inicialmente avaliados de forma descritiva, para a qual foram calculadas 


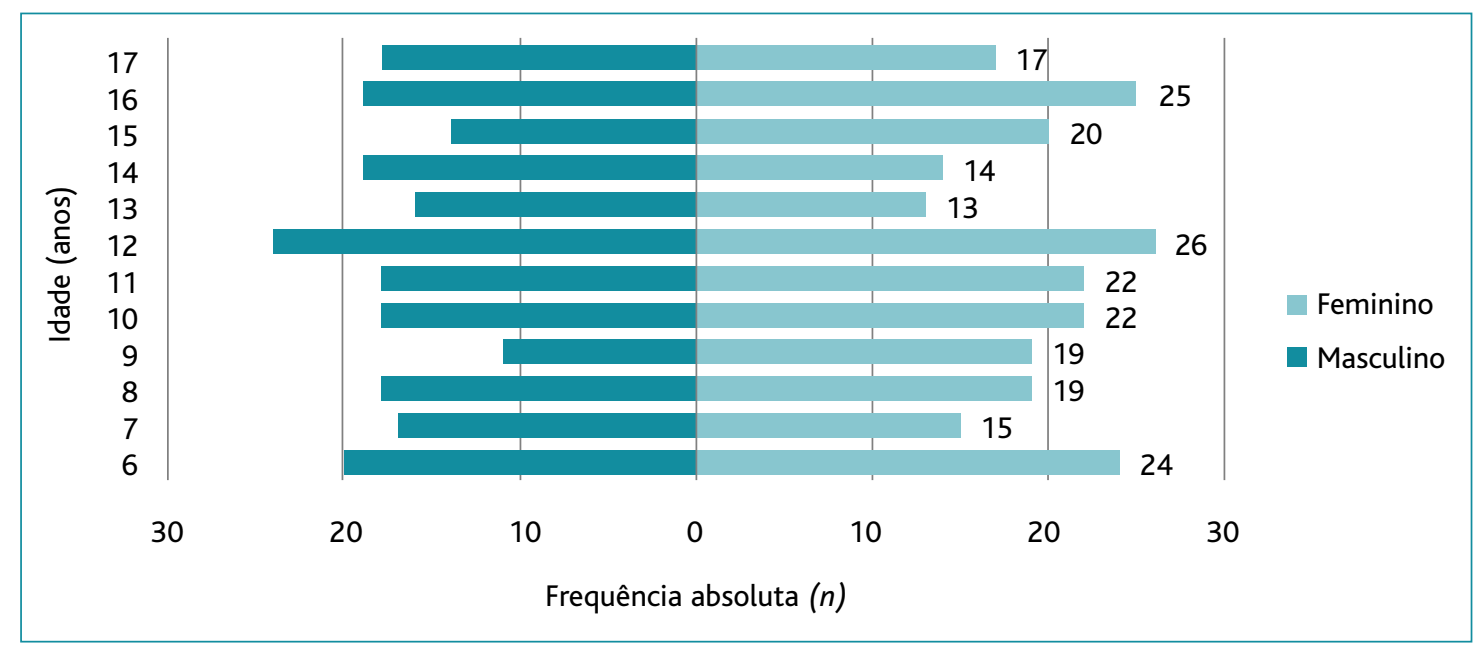

Figura 1. Distribuição etária e por género da amostra.

estatísticas adequadas (média, mediana, frequências absolutas e relativas). Para além desta análise recorreu-se ao teste do Qui-Quadrado para avaliar a independência de variáveis qualitativas nominais e ao teste do $t$-Student para avaliar diferenças entre as médias de dois grupos independentes de variáveis quantitativas. A normalidade foi testada através do teste de Kolmogorov-Smirnov.

O nível de significância usado em toda a análise estatística foi de $5 \%$.

O software usado no tratamento de dados foi o SPSS ${ }^{\circledast}$ v. 25.0, o Microsoft Office Excel ${ }^{\circledR}$ e o Sample Size Calculator by Raosoft ${ }^{\oplus}$.

\section{RESULTADOS}

A amostra foi constituída por 448 crianças e adolescentes. A média de idades foi de 11,48 anos e a mediana foi de 12 anos. Da amostra fizeram parte 236 (52,7\%) rapazes e $212(47,3 \%)$ raparigas, distribuídos por idade e por sexo (Figura 1).

Associando o IMC com os percentis definidos pela $\mathrm{OMS}^{4,12}$ verificou-se que $16,7 \%(n=75)$ das crianças e adolescentes tinham excesso de peso e que $10,7 \%$ $(n=48)$ eram obesas, o que perfaz uma prevalência de peso excessivo (excesso de peso e obesidade) de $37,4 \%$. Apenas $4 \%(n=18)$ das crianças e adolescentes tinham baixo peso (Figura 2).

Quanto à distribuição por género, observou-se uma maior prevalência de excesso de peso no sexo femini- no ( $n=43,57,3 \%)$, enquanto a obesidade foi ligeiramente mais prevalente no sexo masculino ( $n=25,52 \%)$ (Tabelas 1 e 2). No entanto, não foram encontradas diferenças estatisticamente significativas entre géneros relativamente ao peso excessivo $\left(\mathrm{X}^{2}=0,065, p=0,798\right)$. Também não foram encontradas diferenças estatisticamente significativas entre o género e a categoria de peso $\left(\mathrm{X}^{2}=1,579, p=0,664\right)$.

$\mathrm{Na}$ amostra em estudo a idade com maior prevalência de peso excessivo foram os sete anos, com 40,6\% dessas crianças com IMC acima do P85. Em contrapartida, aos seis anos foi onde se observou a menor prevalência de peso excessivo (13,6\%) (Figura 3). No entanto, não foram encontradas diferenças estatisticamente significativas entre idades relativamente ao peso excessivo $(t=0,542, p=0,588)$.

Considerando apenas a idade, observou-se uma maior tendência para o excesso de peso aos sete anos e para a obesidade aos 12 anos.

\section{DISCUSSÃO E CONCLUSÃO}

De acordo com os percentis de IMC definidos pela OMS, ${ }^{4,12}$ verificou-se uma prevalência de $16,7 \%$ de excesso de peso e uma prevalência de $10,7 \%$ de obesidade nas crianças e adolescentes, o que perfez uma prevalência de peso excessivo (excesso de peso e obesidade) de $37,4 \%$ ( IC $\left._{95 \%} 33,76-41,04 \%\right)$. Apesar de não existirem na literatura dados comparativos para a faixa etária da amostra em estudo [6-17 anos], a prevalência 


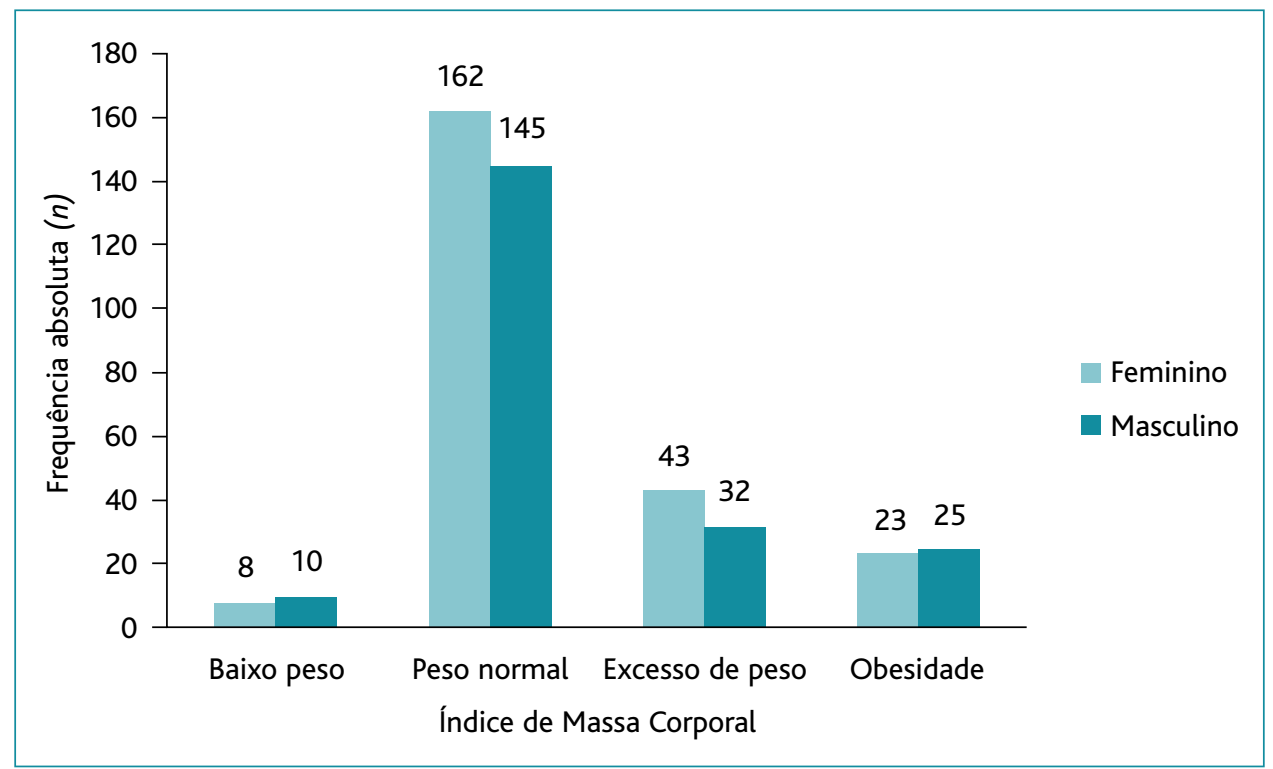

Figura 2. Distribuição da amostra segundo as classes de IMC e género.

\begin{tabular}{|c|c|c|c|c|c|}
\hline \multirow{2}{*}{$\begin{array}{l}\text { Sexo Feminino } \\
\text { Idade }\end{array}$} & \multicolumn{5}{|c|}{ Percentil } \\
\hline & $<3$ & 3 a 85 & 85 a 97 & $>97$ & Total \\
\hline 6 & 1 & 19 & 2 & 2 & 24 \\
\hline 7 & 0 & 8 & 6 & 1 & 15 \\
\hline 8 & 0 & 13 & 4 & 2 & 19 \\
\hline 9 & 0 & 13 & 3 & 3 & 19 \\
\hline 10 & 1 & 15 & 5 & 1 & 22 \\
\hline 11 & 0 & 16 & 3 & 3 & 22 \\
\hline 12 & 1 & 20 & 2 & 3 & 26 \\
\hline 13 & 1 & 11 & 1 & 0 & 13 \\
\hline 14 & 0 & 10 & 4 & 0 & 14 \\
\hline 15 & 2 & 11 & 4 & 3 & 20 \\
\hline 16 & 1 & 17 & 6 & 1 & 25 \\
\hline 17 & 1 & 9 & 3 & 4 & 17 \\
\hline Total & 8 & 162 & 43 & 23 & 236 \\
\hline
\end{tabular}

de peso excessivo foi significativa, perfazendo mais de um terço da amostra.

A prevalência do peso excessivo na faixa etária inferior aos 10 anos foi superior na presente amostra face

\begin{tabular}{|c|c|c|c|c|c|}
\hline \multicolumn{6}{|c|}{$\begin{array}{l}\text { TABELA 2. Distribuição da amostra por percentil e } \\
\text { idade no sexo masculino }\end{array}$} \\
\hline \multirow{2}{*}{$\frac{\text { Sexo Feminino }}{\text { Idade }}$} & \multicolumn{5}{|c|}{ Percentil } \\
\hline & $<3$ & 3 a 85 & 85 a 97 & $>97$ & Total \\
\hline 6 & 1 & 17 & 2 & 0 & 20 \\
\hline 7 & 0 & 11 & 3 & 3 & 17 \\
\hline 8 & 1 & 11 & 4 & 2 & 18 \\
\hline 9 & 1 & 7 & 2 & 1 & 11 \\
\hline 10 & 0 & 10 & 2 & 6 & 18 \\
\hline 11 & 1 & 10 & 4 & 3 & 18 \\
\hline 12 & 1 & 13 & 5 & 5 & 24 \\
\hline 13 & 1 & 10 & 4 & 1 & 16 \\
\hline 14 & 1 & 16 & 1 & 1 & 19 \\
\hline 15 & 1 & 11 & 2 & 0 & 14 \\
\hline 16 & 0 & 16 & 1 & 2 & 19 \\
\hline 17 & 2 & 13 & 2 & 1 & 18 \\
\hline Total & 10 & 145 & 32 & 25 & 212 \\
\hline
\end{tabular}

aos dados disponíveis no Programa Nacional para a Promoção da Alimentação Saudável 2017. ${ }^{6}$ Verificou-se um incremento de $0,9 \%$ para o excesso de peso $(18,2 \%$ vs $17,3 \%)$ e de $2,1 \%$ para a obesidade $(9,8 \%$ vs $7,7 \%)$. 


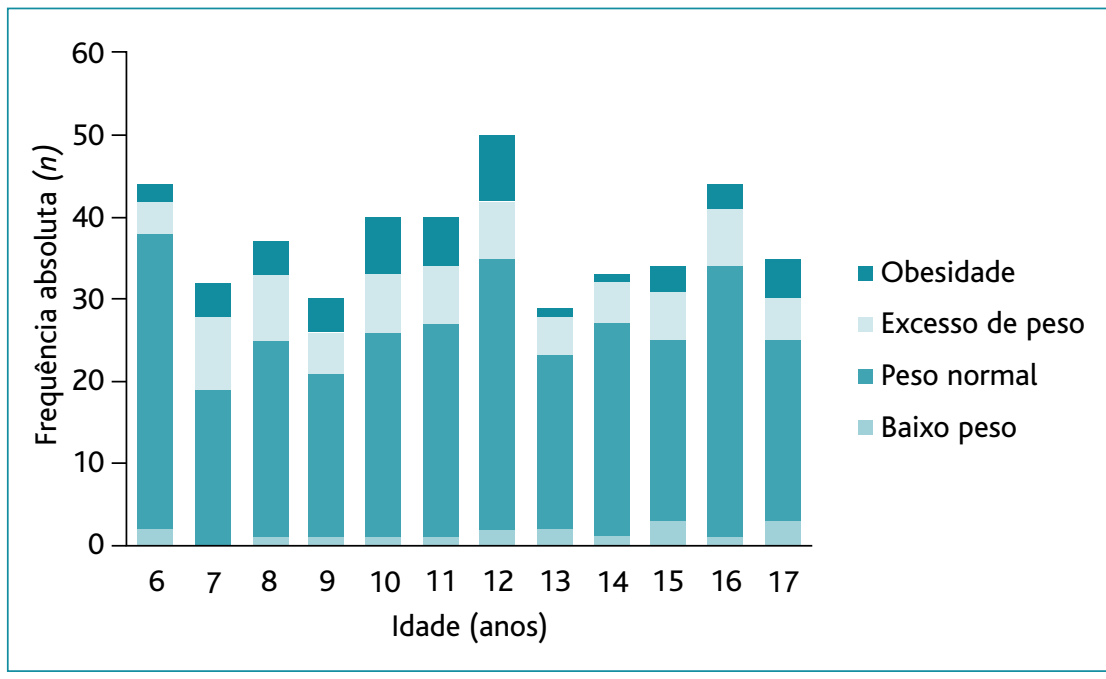

Figura 3. Distribuição da amostra segundo as categorias de IMC e idade.

Na faixa etária dos 10 aos 17 anos, a prevalência do peso excessivo foi inferior aos dados disponíveis no Programa Nacional para a Promoção da Alimentação Saudável 2017. ${ }^{6}$ Verificou-se que o excesso de peso foi inferior na presente amostra $(16,1 \%$ vs $23,6 \%)$, enquanto a prevalência de obesidade foi superior $(11,1 \%$ vs $8,7 \%$ ).

Comparativamente aos dados regionais do Alentejo obtidos na quinta ronda do COSI Portugal (2019) ${ }^{13} \mathrm{em}$ crianças dos seis aos oito anos, a presente amostra apresenta uma menor prevalência de excesso de peso $(18,6 \%$ vs $23,9 \%)$ e obesidade ( $8,8 \%$ vs $9,7 \%)$.

Pela análise dos dados pôde ainda concluir-se que se trata de um problema transversal a todas as idades, não havendo diferenças estatisticamente significativas entre idades relativamente ao peso excessivo.

Em relação à variabilidade dependente do género pôde concluir-se pela análise dos dados que a prevalência de excesso de peso foi superior no sexo feminino $(18,2 \%)$, a prevalência de obesidade foi superior no sexo masculino $(11,8 \%)$ e a prevalência de peso excessivo foi superior no sexo feminino ( $28 \%$ vs $26,9 \%$ ). No entanto, não foram encontradas diferenças estatisticamente significativas entre géneros relativamente ao peso excessivo, nem entre género e cada categoria de peso.

Se se considerar a faixa etária dos seis aos oito anos, por forma a estabelecer comparação com a quinta ronda do COSI Portugal, ${ }^{13}$ verifica-se que a prevalência de excesso de peso é superior nas raparigas $(10,6 \%)$ e que a prevalência de obesidade é igual em ambos os géneros $(4,4 \%)$. Estes resultados não estão de acordo com os resultados obtidos no referido estudo, onde as prevalências de excesso de peso e de obesidade são superiores no sexo masculino, ${ }^{13} \mathrm{o}$ que pode dever-se ao número limitado de indivíduos (113) na amostra considerada.

Em relação ao baixo peso, entre os seis e os oito anos, a prevalência obtida foi de $0,7 \%$, inferior à prevalência obtida nos resultados da quinta ronda do COSI Portugal de $1,3 \% \mathrm{e}$ do Alentejo $(0,9 \%) \cdot{ }^{13}$ A prevalência do baixo peso nesta faixa etária foi superior no sexo masculino; no entanto, o número de casos foi muito limitado, pelo que não se podem estabelecer comparações exatas com os resultados obtidos no referido estudo.

Como limitações destaca-se o facto de a obtenção de dados antropométricos ser adquirida por diferentes enfermeiros, no âmbito da consulta de enfermagem que precede a consulta médica de saúde infantil e juvenil. Há que considerar a variabilidade interobservador, apesar dos métodos de colheita de dados terem sido idênticos. ${ }^{2,4}$ Por outro lado, a seleção da amostra foi condicionada pelas crianças frequentadoras da consulta de saúde infantil e juvenil entre janeiro de 2017 e outubro de 2018, o que excluiu um número significativo de indivíduos.

Os resultados do presente estudo comprovam que a obesidade infantil constitui um problema de saúde muito prevalente no concelho de Portalegre, à semelhança do que se verifica a nível nacional. A sua importância advém do facto de afetar a saúde das crianças, constituindo a prevenção e o diagnóstico precoce os pilares para o seu combate.

Este estudo poderá constituir o ponto de partida para o desenvolvimento de uma intervenção na comunidade para minimizar este problema e os efeitos em saúde que dele advêm, ${ }^{15-23}$ nomeadamente mediante a sensibilização da comunidade escolar para o problema da obesidade infantil em parceria com a equipa de saúde escolar. 


\section{AGRADECIMENTOS}

Os autores agradecem a Amílcar Pires e ao Dr. Oleh Yaremiy pelo auxílio na recolha de dados dos utentes inscritos na Unidade de Saúde Familiar. Agradecem ainda à Dra. Adília Oliveira pelo apoio na análise estatística dos dados.

\section{REFERÊNCIAS BIBLIOGRÁFICAS}

1. Carmo I, Santos O, Camolas J, Vieira J, Carreira M, Medina L, et al. Overweight and obesity in Portugal: national prevalence in 2003-2005. Obes Rev. 2008;9(1):11-9.

2. Rito A, Breda J, Carmo I, editors. Guia de avaliação do estado nutricional infantil e juvenil [Internet]. Lisboa: Direção-Geral da Saúde/Instituto Nacional de Saúde Dr Ricardo Jorge; 2010. Available from: http:// www2.insa.pt/sites/INSA/Portugues/Publicacoes/Outros/Documents/ AlimentacaoNutricao/GuiaAvaliacaoEstadoNutricional.pdf

3. Direção-Geral da Saúde. Consórcio europeu lança projeto para combater a obesidade infantil na Europa [homepage]. Lisboa: DGS; 2018 [cited 2018 Jun 18]. Available from: https://www.dgs.pt/em-destaque/lancado-projeto-de-10-milhoes-de-euros-para-combater-a-obesidade-infantil-na-europa.aspx

4. Direção-Geral da Saúde. Programa nacional de saúde infantil e juvenil: norma n. ${ }^{\circ}$ 010/2013, de 31/05/2013. Lisboa: DGS; 2013.

5. Direção-Geral da Saúde. Plano nacional de saúde: revisão e extensão a 2020 [Internet]. Lisboa: DGS; 2015.Available from: http://pns.dgs.pt/files/2015/06/Plano-Nacional-de-Saude-Revisao-e-Extensao-a2020.pdf.pdf

6. Direção-Geral da Saúde. Programa nacional para a promoção da alimentação saudável [homepage]. Lisboa: DGS; 2017. Available from: http://www.alimentacaosaudavel.dgs.pt/pnpas/

7. Filipe J, Godinho CA, Graça P. Intervenções comportamentais de prevenção da obesidade infantil: estado da arte em Portugal [Behavioural interventions for childhood obesity prevention: state of the art in Portugal]. Psychol Commun Health. 2016;5(2):170-84. Portuguese

8. Camolas J, Santos O, Moreira P, Carmo I. Promovendo mudanças comportamentais sustentaveis no controlo do peso corporal [Promoting sustainable behavior change in body weight control]. Acta Med Port. 2014;27(1):99-107. Portuguese

9. World Health Organization. Obesity: preventing and managing the global epidemic [homepage]. Geneva: WHO; 2000. Available from: https://apps.who.int/iris/handle/10665/42330

10. World Health Organization. European food and nutrition action plan 2015-2020 [Internet]. Copenhagen:WHO Regional Office for Europe; 2015.Available from:http://www.euro.who.int/_data/assets/pdf_file/ 0003/294474/European-Food-Nutrition-Action-Plan-20152020en.pdf?ua $=1$

11. de Onis M, Lobstein T. Defining obesity risk status in the general childhood population: which cut-offs should we use? Int J Pediatr Obes. 2010;5(6):458-60.

12. World Health Organization. Growth reference data for 5-19 years [ho- mepage]. Geneva:WHO; 2020. Available from: https://www.who.int/ toolkits/growth-reference-data-for-5to19-years

13. Rito A, Sousa RC, Mendes S, Graça P. Childhood Obesity Surveillance Initiative: COSI Portugal 2019. Lisboa: Instituto Nacional de Saúde Dr. Ricardo Jorge; 2020.Available from: https://alimentacaosaudavel.dgs.pt/ activeapp2020/wp-content/uploads/2020/01/COSI2019_FactSheet .pdf

14. Rito A, Sousa RC, Mendes S, Graça P. Childhood Obesity Surveillance Initiative: COSI Portugal - Relatório 2016 [Internet]. Lisboa: Instituto Nacional de Saúde Dr. Ricardo Jorge; 2017. Available from: http://hdl. handle.net/10400.18/4857

15. Galani $\mathrm{C}$, Schneider H. Prevention and treatment of obesity with lifestyle interventions: review and meta-analysis. Int J Public Heal. 2007; 52(6):348-59.

16. Camolas J, Correia F, Lima Reis J, Moreira P. Medidas terapêuticas: nutrição. In: Galvão-Teles A, Lima Reis J, Dias T, editors. Obesidade, prevenção e terapêutica. Lisboa: Presença; 2008. p. 28-31.

17. Leidy HJ, CampbellWW. The effect of eating frequency on appetite control and food intake: brief synopsis of controlled feeding studies. J Nutr. 2011;141(1):154-7.

18. Hu FB. Resolved: there is sufficient scientific evidence that decreasing sugar-sweetened beverage consumption will reduce the prevalence of obesity and obesity-related diseases. Obes Rev. 2013;14(8):606-19.

19. Garaulet M, Gómez-Abellán P, Alburquerque-Béjar JJ, Lee YC, Ordovás JM, Scheer FA. Timing of food intake predicts weight loss effectiveness. Int J Obes. 2013;37(4):604-11.

20. Lantz $H$, Peltonen $M$, Agren L, Torgerson JS. A dietary and behavioural programme for the treatment of obesity: a 4-year clinical trial and a long-term posttreatment follow-up. J Intern Med. 2003;254(3):272-9.

21. Rolls BJ. What is the role of portion control in weight management? Int J Obes. 2014;38 Suppl 1:S1-8.

22. Elfhag K, Rössner $\mathrm{S}$. Who succeeds in maintaining weight loss? A conceptual review of factors associated with weight loss maintenance and weight regain. Obes Rev. 2005;6(1):67-85.

23. Buckland G, Bach A, Serra-Majem L. Obesity and the Mediterranean diet: a systematic review of observational and intervention studies. Obes Rev. 2008;9(6):582-93.

\section{CONFLITO DE INTERESSES}

Os autores declaram não possuir quaisquer conflitos de interesse.

\section{ENDEREÇO PARA CORRESPONDÊNCIA}

Adriana Gameiro

E-mail: adrianagameiromgf@gmail.com

https://orcid.org/0000-0002-1652-3266

Recebido em 25-05-2020

Aceite para publicação em 10-03-2021 


\section{ABSTRACT}

\section{CHILD NUTRITIONAL STATUS IN THE COUNTY OF PORTALEGRE}

Objectives: To determine the prevalence of low weight, normal weight, overweight, and obesity among the school-age population in a Family Health Care Unit (USF) in the municipality of Portalegre and to determine whether there is a statistically significant difference between gender and weight, and between age and weight.

Type of study: Cross-sectional, descriptive, and analytical observational study.

Location: USF Portus Alacer, municipality of Portalegre.

Population: USF Portus Alacer users [6-17 years old].

Methods: Determination of the body mass index (BMI) of 448 children and adolescents [6-17 years old], surveyed at USF Portus Alacer between January 2017 and October 2018 [95\% confidence interval (Cl), 3.64\% margin of error]. The participants were classified into four categories according to the BMI cut-off points established by the WHO for age and gender. The software used for data processing was SPSS ${ }^{\circledR}$ v. 25.0, Microsoft Office Excel ${ }^{\circledR}$, and Sample Size Calculator by Raosoft ${ }^{\circledR}$. The statistical tools used were the Chi-square test and the t-Student test. Normality was tested using the Kolmogorov-Smirnov test. Results: Of the children evaluated, $52.7 \%$ were male. The median age was 12 years old. Excessive weight prevalence was $37.4 \%$. The prevalence of overweight was $16.7 \%$, higher in females, and obesity was $10.7 \%$, slightly higher in males. The highest prevalence of overweight was observed at age seven. No statistically significant differences were found between gender and weight category, nor between ages regarding excessive weight.

Conclusions: Despite the decrease in the prevalence of childhood obesity and overweight in Portugal in recent years, these entities represent a serious public health problem. In the municipality of Portalegre, the prevalence of excessive weight in children under 10 years old was higher compared to the data available in the literature. From 10 to 17 years old, the prevalence was lower.

Keywords: Overweight; Pediatric obesity; Body mass index. 